\title{
Análisis de la percepción del alumnado ante la metodología de trabajar en grupos tutorizados
}

Eva Serna ${ }^{\mathrm{a}}$, Teresa San Miguel ${ }^{\mathrm{a}}$, Javier Megías ${ }^{\mathrm{a}}$, y María Dolores Mauriciob.

${ }^{a}$ Departamento de Patología, Universitat de València. Eva.serna@uv.es ${ }^{\mathrm{b}}$ Departamento de Fisiología, Universitat de València.m.dolores.mauricio@uv.es

\begin{abstract}
The main objective of this paper is to analyze the opinion and perspective that the students have in view of the methodology of working in tutored groups. This methodology adopted in the teaching-learning process is important for the development of competences. The ability to work in a team and the ability to communicate are very significant transversal competences that have a great impact or potential in student learning. This paper presents an analysis of student assessments collected through anonymous opinions after the elaboration of a tutorial work carried out in a group in the Medicine Degree of the University of Valencia during the years 2015-2016 and 20162017. It is essential to take into account the opinion of the students to adopt measures of teacher improvement, correct or modify the methodology and solve situations arising in the real learning environment. Our conclusion is the students' perspective is very positive and they like the methodology of working in tutored groups.
\end{abstract}

Keywords: Teamwork, turored groups, competences, university teaching

\section{Resumen}

El objetivo de este trabajo es analizar la opinión y perspectiva que tiene el alumnado ante la metodología de trabajar en grupos tutorizados. Esta metodología adoptada en el proceso de enseñanza-aprendizaje es importante para el desarrollo de competencias. La capacidad de trabajo en equipo y la capacidad para comunicarse son competencias transversales muy significativas y que tienen un gran impacto o potencial en el aprendizaje del alumno. Se presenta en este trabajo un análisis de las valoraciones del alumnado recogidas mediante opiniones anónimas después de la elaboración de un trabajo tutorizado realizado en grupo en el Grado de Medicina de la Universitat de València durante los cursos 2015-2016 y 2016-2017. Es fundamental tener en cuenta la opinión del alumnado para adoptar medidas de mejora docente, corregir o modificar la metodología y resolver situaciones surgidas en el entorno real de aprendizaje. Nuestra conclusión es que la perspectiva del alumnado es muy positiva que les gusta la metodología de trabajar en grupos tutorizados.

Palabras clave: Trabajo en equipo, grupo tutorizado, competencias, educación universitaria 


\section{Introducción}

Las universidades españolas se encuentran inmersas en el Espacio Europeo de Educación Superior (EEES) (Declaración de Bolonia, 1999). La adecuación al EEES ha supuesto una profunda renovación en la estructura curricular, las estrategias de aprendizaje y modelos de evaluación. Los pilares metodologógicos y pedagógicos de este nuevo de modelo vienen marcados por un lado, el alumno es el centro de su propio aprendizaje dotándolo de las herramientas necesarias (diferente papel del docente, (Fernández, 2006) y permitiéndole un aprendizaje más autonomo, efectivo, creativo y activo (Salaburu, 2011; González y Wagenaar, 2006; Gijon y Crisol, 2012). Y por otro, en cambios en la organización de los aprendizajes como es el aprendizaje en grupo que precisa de un seguimiento del trabajo realizado (Zabalza, 2003) y que crea una interdependencia positiva. (Ovejero, 1990; Jonson y Jonson 1999).

En este proceso adquiere gran importancia el aprendizaje por competencias. Por ello, se requieren de nuevas metodologías docentes que ayuden y contribuyan al desarrollo integrado de competencias. El hecho de incluir este término a los currículos universitarios permite dar una formación más completa y real al alumnado ya que se integrarían los componentes personales (conocimientos, habilidades cognitivas, motivación, actitudes, emociones), componentes sociales y de conductas (acciones, comportamientos, iniciativas) guiadas por unos fines u objetivos. El hecho de trabajar por competencias hace que la posterior incorporación al mundo laboral del alumnado sea mejor.

Dentro de este contexto el Grado de Medicina tiene unas competencias básicas, generales, transversales y específicas que ha de cumplir un alumno cuando acaba su etapa de formación. Dentro de las competencias transversales más significativas están la capacidad para trabajar en equipo y para relacionarse con otras personas del mismo o distinto ámbito profesional así como la competencia de tener la capacidad para comunicarse. Dentro de las competencias específicas destacamos:

1.Conocer,valorar críticamente y saber utilizar las tecnologías y fuentes de información clínica y biomédica, para obtener, organizar, interpretar y comunicar información clínica, científica y sanitaria.

2.Comprender e interpretar críticamente textos científicos.

3.Conocer los principios del método científico, la investigación biomédica y el ensayo clínico.

4.Saber realizar una exposición en público, oral y escrita, de trabajos científicos y/o informes profesionales.

Nuestra hipótesis es que este tipo de metodología propuesta mediante el desarrollo de un trabajo en equipo tutorizado por el docente permitiría desarrollar y adquirir las competencias propuestas. Para ello, es necesario acercar la opinión del alumnado al docente sobre su experiencia con este tipo de metodología de trabajo y así poder adoptar medidas de mejora docente, corregir o modificar la metodología y resolver situaciones surgidas en el entorno real de aprendizaje.

(c)) BY-NC-ND 2017, Universitat Politècnica de València 


\section{Objetivos}

El objetivo fundamental del presente trabajo es analizar la percepción del alumnado sobre el desarrollo de un tema tutorizado trabajando en grupo.

Para ello los objetivos específicos son:

1- Analizar las valoraciones positivas y negativas desde el punto de vista del alumnado.

2- Desarrollar estrategias de mejora por parte del equipo docente.

\section{Descripción de la metodología docente:}

La experiencia se llevó a cabo con el alumnado matriculado en la asignatura de Biología de $1^{\text {er }}$ Curso en los grupos ARA y V, del Grado de Medicina de la Universitat de València en el curso académico 2015-2016 y 2016-2017 (Código de Asignatura: 34446 con 6 ECTS y con carácter de formación básica). El número total de alumnos que participaron en el feedback al docente fue de 11 de un total de 32 en el curso 2015-16 y 25 de un total 26 alumnos en el curso 2016-2017.

Los trabajos tutorizados para los dos cursos se plantearon de la misma forma. Grupos con un número reducido de estudiantes (4-5 personas). El tema que se designaba a cada equipo era una enfermedad no muy común que tenían que investigar realizando una búsqueda bibliográfica, centrándose en los aspectos desde el punto de vista de la biología celular que fallaban o se alteraban y desencadenaban dicha patología. Cabe destacar que todo el marco teórico de la biología celular se ha dado en las cláses teóricas de la asignatura. Finalmente, el grupo tiene que realizar un póster sintetizando la información encontrada y exponerla a todos los demás grupos.

Para ello, el contenido del trabajo sigue el esquema que muestra la Figura 1 que les sirve de orientación y está basado en el método científico. 


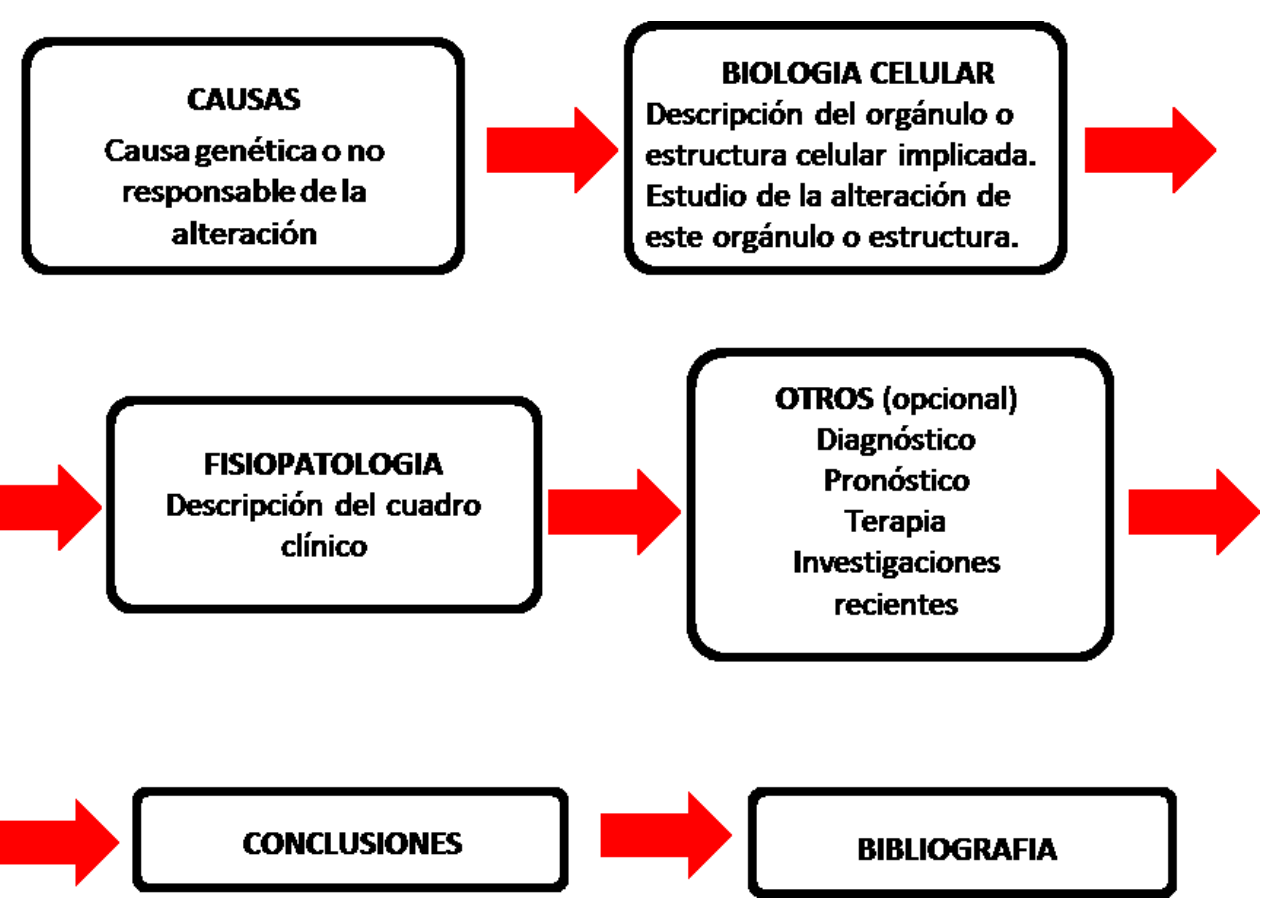

Figura 1. Esquema del contenido del trabajo tutorizado.

Primeramente, describir la enfermedad, el orgánulo o componente celular responsable del desarrollo de la patología. En concreto, elegimos enfermedades raras que son enfermedades que no van a profundizar mucho en el desarrollo de su Grado, para que sea un tema atractivo y curioso. Después, profundizar en la causa (genética o no) responsable de la alteración.

De ahí pasar de manera muy general a la Fisiopatología de la enfermedad y dependiendo de la información encontrada y de la patología en cuestión, profundizar en aspectos como diagnóstico, pronóstico, terapia e investigaciones recientes. El equipo docente remarcamos como muy importante la investigación y publicaciones científicas actuales que encuentren de su tema en concreto.

Como apartado final realizar unas conclusiones del trabajo y especificar de manera formal la bibliografía empleada. 
Los temas planteados fueron:

Tabla 1. Temas designados a cada grupo

\begin{tabular}{cc}
\hline Biología celular & Patología asociada \\
\hline Uniones tipo GAP & Síndrome de Vohwinkel \\
Membrana & Esferocitosis hereditaria \\
plasmática & \\
Desmosoma & Pénfigo vulgar \\
Retículo & Pseudoacondroplasia \\
endoplásmico & \\
Lisosoma & Enfermedad de Pompe \\
Mitocondria & MERRF
\end{tabular}

El esquema de la metodología docente empleada generando una dinámica de grupo se ilustra en la siguiente Figura 2.

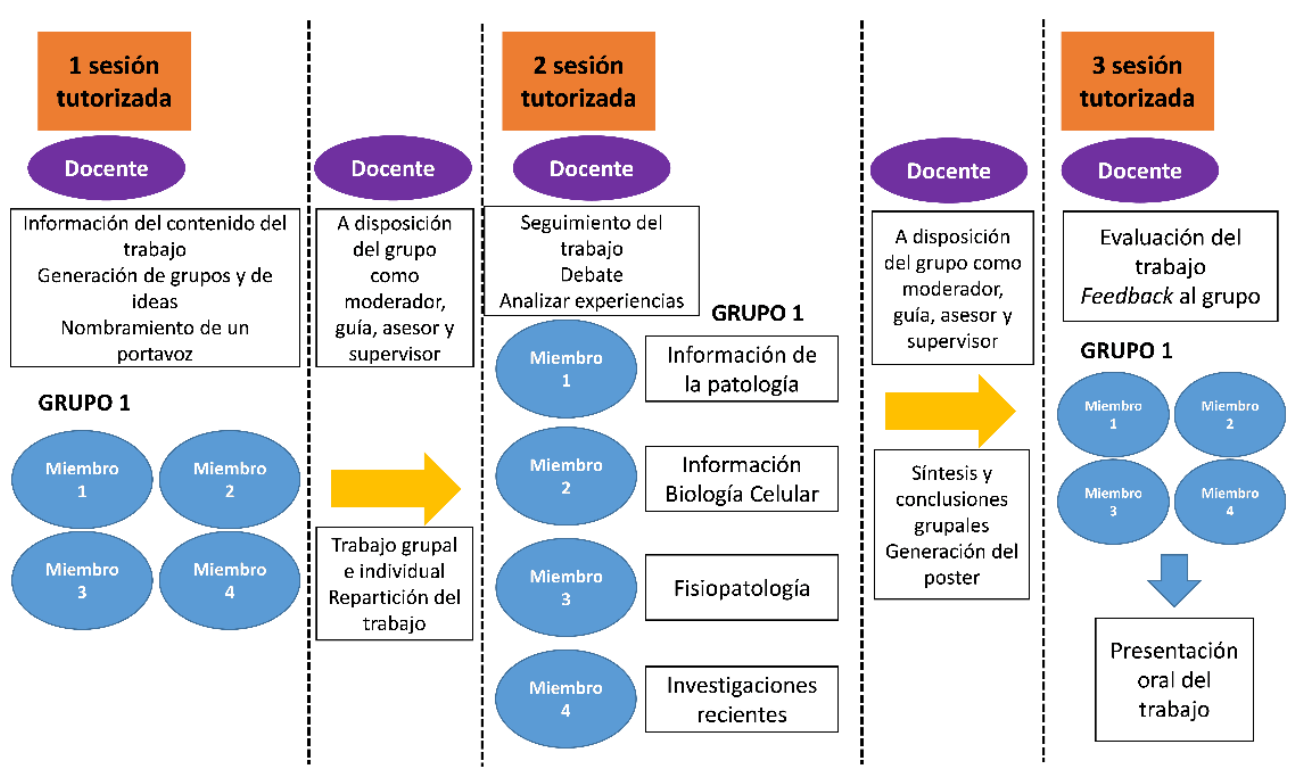

Figura 2. Metodología de trabajo en grupo empleada. 
La metodología docente consiste en 3 sesiones tutorizadas. En la primera se informa a los alumnos sobre el procedimiento de trabajo, se generan los grupos y se elige al portavoz de cada equipo. A partir de este momento, los estudiantes empiezan a trabajar y se reparten el contenido. En todo momento el equipo docente está como moderador, guía, asesor y supervisor. La segunda sesión con el docente consiste en compartir su experiencia grupal hasta ese momento, detectar posibles conflictos, favorecer el debate y corroborar que el trabajo se está desarrollando de forma correcta. En la tercera y última sesión se presenta oralmente el trabajo y el docente evalúa el resultado final compartiendo su perspectiva con el alumnado.

Después de la exposición del trabajo grupal se les invita a dar un feedback al docente sobre los aspectos positivos y negativos que ha encontrado cada alumno de manera individual en el desarrollo del trabajo en equipo de manera anónima.

Este trabajo es evaluado sobre 1 punto sobre un total de 4 puntos de la parte de Evaluación Práctica de la asignatura de Biología siguiendo una rúbrica diseñada específicamente para evaluar tanto el proceso como el resultado final.

\section{Resultados}

Los resultados que obtenemos tras el análisis de las valoraciones del alumnado se pueden dividir en, por una lado, valoraciones positivas (Figura 3) y, por el otro, valoraciones negativas (Figura 4) para las dos anualidades estudiadas. Cabe destacar que los resultados se han porcentuado al total de las contribuciones del alumnado obtenidas para cada anualidad.

Si profundizamos en detalle en las valoraciones positivas (Figura 3), en primer lugar, utilizan mucho el verbo "aprender" en cualquiera de las contribuciones que nos devuelven, ya sea en el curso 2015-2016 como para el 2016-2017. Siendo el porcentaje más elevado para las dos anualidades (aproximadamente 91\% para 2015-2016 y un 52\% para 20162017). Es la contribución mejor valorada por el alumnado en general. 


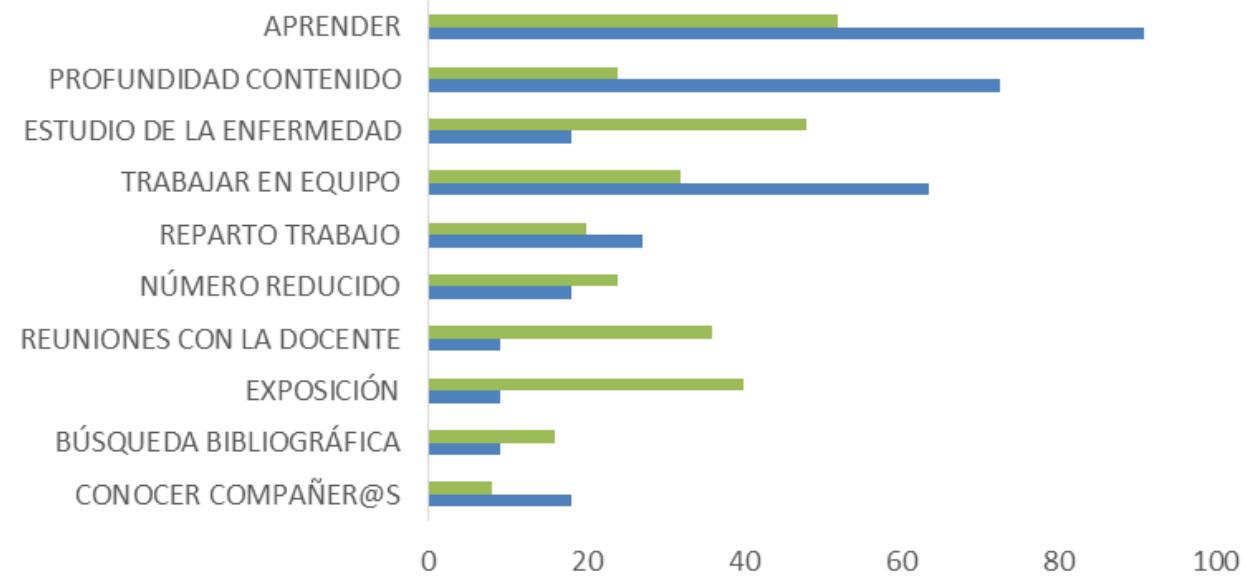

Figura 3. Valoraciones positivas del alumnado frente al grupo tutorizado.

En segundo lugar, coinciden también los dos cursos que el contenido y temas planteados son valoradas positivamente, aunque en 2015-2016 destacan más la profundidad del contenido (72\%) y en 2016-2017 el estudio de la enfermedad (48\%).

Existe también una coincidencia en valores de porcentaje para los dos cursos cuando hablan de reparto del trabajo (27\% en $2015-2016$ y $20 \%$ en $2016-2017)$ y en trabajar con un número reducido de personas (18\% en 2015-2016 y 24\% en 2016-2017).

El trabajar en equipo lo destacan el 64\% en 2015-2016 y el 32\% en 2016-2017.

En 2016-2017 se destaca un aumento de contribuciones haciendo referencia a las reuniones planteadas por el docente (36\%) y a la exposición (40\%). En 2015-2016 se detecta un aumento de contribuciones que son importantes en el aspecto de conocer a compañeros $(18 \%)$. Y en las dos anualidades también valoran la búsqueda de referencias bibliográficas ( $9 \%$ en $2015-2016$ y $16 \%$ en 2016-2017).

Si profundizamos en el análisis de las valoraciones negativas (Figura 4) coindiden los dos cursos en destacar que trabajar en equipo es difícil, siendo la contribución más elevada dentro de las negativas (36\% en 2015-2016 y 32\% en 2016-2017). Además este tipo de trabajo lo consideran una pérdida de tiempo (27\% en 2015-2016 y 28\% en 2016-2017). 


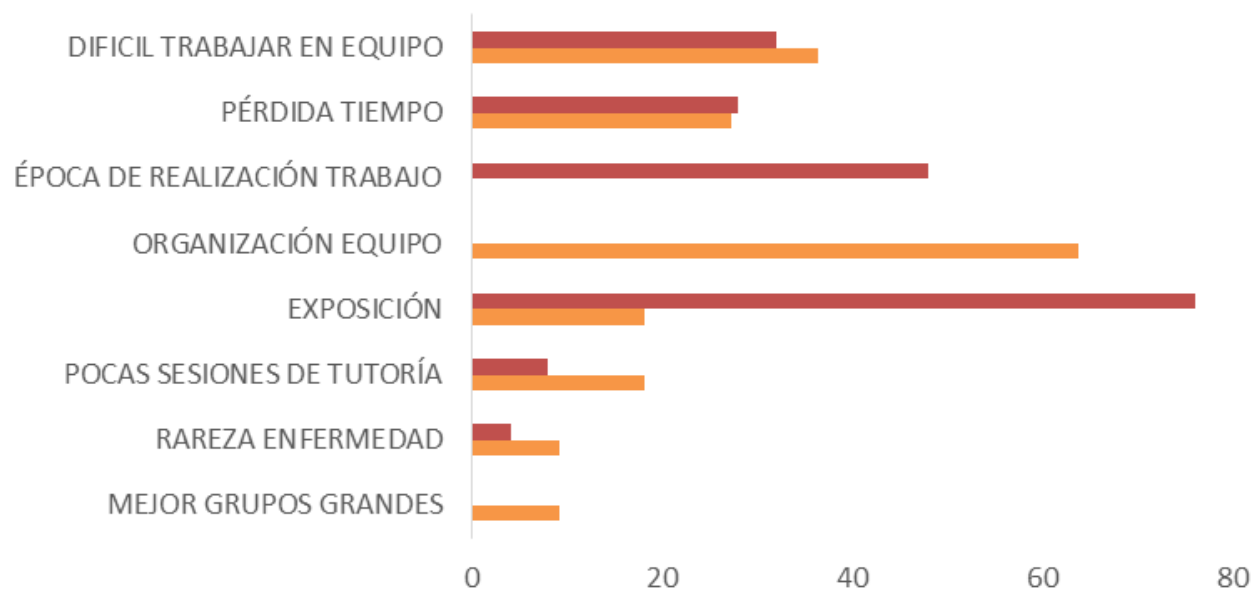

Figura 4. Valoraciones negativas del alumnado frente al grupo tutorizado.

En 2015-2016 consideraron un aspecto negativo la organización del equipo, ya que destacaban difícil cuadrar agendas con horarios. Mientras que en el curso 2016-2017 lo más destacable negativamente fue la época de realización del trabajo.

La exposición fue destacada muy positivamente para un gran grupo de alumnos pero también hay un porcentaje de alumnado que lo valoran negativamente sobre todo por el tema de las vergüenzas (2015-2016 fue 18\% mientras que en 2016-2017 llegó a tener un porcentaje del $76 \%$ ).

Valoraciones negativas de menor relevancia fueron que creen necesitar más sesiones de tutoría y el tema que proponemos de la enfermedad no es muy común. Así como hay gente que prefiere trabajar con grupos más grandes.

\section{Conclusiones}

En la guía docente de la asignatura de Biología se tiene como uno de los objetivos de resultados de aprendizaje la de adquirir "capacidad de trabajo en equipo y habilidades para el desarrollo y exposición de temas de Biología Celular”. Es en este contexto donde se desarrolla este trabajo tutorizado.

Para fomentar esta competencia universitaria creamos grupos reducidos y dinámicos en un entorno colectivo estructurado en la que el docente actua de mediador, moderador, guía, asesor y supervisor, y además planteamos un tema atractivo para fomentar la motivación.

$\mathrm{Si}$ profundizamos en detalle en las opiniones del alumnado encontramos resultados sorprendentes que iremos discutiendo a continuación.

En primer lugar, nos llama la atención que este trabajo lo valoren con una palabra “APRENDER". Este verbo está presente en muchos alumnos/as siendo el mejor valorado tanto para la anualidad 2015-2016 como para la anualidad 2016-2017 y, por tanto,

(cc)) EY-NC-ND 2017, Universitat Politècnica de València 
coinciden que con este trabajo aprenden. Comentarios como "aprender a aceptar que tus propuestas no siempre pueden tener éxito", "aprender a realizar investigaciones más exhaustivas y con mejores fuentes que nos servirá para trabajos futuros", "aprender a trabajar en grupo y convivir con ellos además de que te aporten una serie de conocimientos o ideas creativas muy interesantes", "conocer a gente nueva y nuevos enfoques y formas de trabajar. Aprender de los demás", "aprender conceptos muy específicos sobre los diferentes orgánulos", "se aprende de una forma más dinámica", "aprender a hablar en público", "aprender a buscar en páginas de webs científicas", "aprender todo el trabajo y profundizar más sobre la enfermedad"...son algunos de los ejemplos.

Sobre todo porque es justamente un pilar de los que comentábamos por el que apuesta la nueva reforma metodológica en la que el docente es un guía para el alumno y le ayuda a construir su propio aprendizaje.

Además afirman que les parece interesante el tema del trabajo con comentarios como "enfermedad relacionada con la materia", "el conocimiento que he extraido del trabajo", "todo lo que sé ahora acerca de la enfermedad y de la estructura celular", "me ha parecido muy interesante y agradezco este tipo de oportunidades para aprender", "profundizar en una enfermedad interesante", "me ha gustado el tema del trabajo que trata de enfermedades y son aspectos no vistos en clase", "relación entre la biología y las enfermedades raras muy interesantes "...es decir, los resultados muestran que les gusta este tipo de trabajos, les parece atractivo e integrador con la materia dada en la clase teórica, y es por ello, que se puede extrapolar a una mayor motivación e interés por la asignatura, siendo la motivación el motor fundamental para un aprendizaje activo.

Como valoraciones positivas también coinciden las dos clases que les gusta repartir el trabajo, les gusta trabajar en equipo y de manera cooperativa. Y que además trabajan mejor con un número reducido de integrantes. Sobre todo es destacable que su percepción sea tan positiva aunque opinen en menor medida que es difícil trabajar en equipo y que se pierde mucho tiempo.

Centrándonos en la exposición, podemos decir que la perspectica es buena ya que muchos la califican de positiva pero existe un colectivo que el tema de la vergüenza y los nervios hace que no le gusten este tipo de situaciones. Esto se refleja en sus comentarios "exponer me supone mucho esfuerzo y lo paso realmente mal y más delante de los compañeros", "me ha ayudado a perder la vergüenza en publico", "una ocasión más para hablar en público", "deberiamos exponer la parte que hemos preparado solo", "críticas constructivas de la profesora al final de la exposición", "realizar la exposición delante de la clase es incómodo así como responder a las preguntas", "las preguntas ponen nerviosos a las personas que exponen"....

Además la época en la que se plantea el trabajo también es un hándicap porque la diferencia entre la anualidad 2015-2016 es que el trabajo se planteó el primero de todos los del cuatrimestre, mientras que en la anualidad 2016-2017 fue el último de todas las asignaturas. Es por ello, que en las valoraciones negativas se observe que la época de trabajo sea 
importante para el alumnado ya que tienen más presentes las fechas de examen y su perspectiva sea más negativa a la hora de valorar este tipo de trabajos.

Como resultado final podemos destacar que la perspectiva del alumnado fue muy positiva a este tipo de metodología. Esto hecho contrarresta con las devoluciones verbales que nos dan en clase y durante el desarrollo del trabajo donde tan solo destacan "la pérdida de tiempo" que les causa. Es por ello, que tras el análisis estamos muy motivados para seguir en esta misma línea de trabajo. $\mathrm{Y}$ nos da que pensar que cuando les pedimos que reflexionen sobre su proceso de aprendizaje es cuando emergen las opiniones más sinceras y profundas.

Además el trabajo grupal les ayuda a desarrollar una sensibilización positiva de trabajo con sus iguales y se fomenta el respeto a la cohesión y un clima de cooperación. De manera también directa la responsabilidad de un objetivo final común hace que se generen conflictos, importantes para su desarrollo y crecimiento personal y profesional.

Podemos concluir, por tanto, que no solo aprenden competencias transversales sino que también trabajan competencias específicas como: “conocer, valorar críticamente y saber utilizar las tecnologías y fuentes de información clínica y biomédica, para obtener, organizar, interpretar y comunicar información clínica, científica y sanitaria", "comprender e interpretar críticamente textos científicos", "conocer los principios del método científico, la investigación biomédica y el ensayo clínico" y "saber realizar una exposición en público, oral y escrita, de trabajos científicos y/o informes profesionales".

Con nuestra experiencia de análisis concluimos que este tipo de trabajos tutorizados funcionan, con sus opiniones podemos mejorar la práctica de apoyo al alumno y, por tanto, el aprendizaje. Y cuyas opiniones son mayoritariamente valoradas positivamente, les gusta trabajar en equipo con un apoyo docente.

\section{Referencias}

FERNÁNDEZ MARCH, A. (2006). "Metodologías activas para la formación de competencias". Educatio Siglo XXI, vol 24, p. 35-56

OVEJERO BERNAL, A. (1990). El aprendizaje cooperativo, una alternativa eficaz a la enseñanza tradicional. Barcelona: Promociones y Publicaciones Universitarias.

JONSON, D. W. y JONSON, R. J. (1999). Aprender juntos y solos. Buenos Aires: Grupo Editorial Aique.

GONZÁlEZ, J. y WAGENAAR, R. (eds.). (2006). Tuning Educational Structures in Europe. Informe final. Proyecto piloto, Fase 2. Bilbao: Universidad de Deusto.

GIJÓN, J. y CRISOL, E. (2012).” La internacionalización de la Educación Superior. El caso del Espacio Europeo de Educación Superior". Revista de Docencia Universitaria. REDU. Monográfico: "Buenas prácticas docentes en la enseñanza universitaria". vol 10 (1), p. 349-414.

SALABURU, P. (dir.) (2011). España y el proceso de Bolonia. Un encuentro imprescindible. Madrid: Academia Europea de Ciencias y Artes de España.

ZABALZA, M. A. (2003). Competencias docentes del profesorado universitario. Calidad y desarrollo profesional. Madrid: Narcea.

(c)) BY-NC-ND 2017, Universitat Politècnica de València 